\title{
Radial diffusion of relativistic electrons in Neptune's magnetosphere
}

\author{
R. S. Selesnick and E. C. Stone \\ California Institute of Technology, Pasadena
}

\begin{abstract}
Measurements of $\gtrsim 1 \mathrm{MeV}$ electrons in Neptune's magnetosphere from $\widetilde{L} \approx 4$ to 14 are well represented by solutions of a model radial diffusion equation that includes losses due to absorption by the satellite Proteus. The model provides estimates of the radial diffusion coefficient, which is $5 \times 10^{-8}(L / 5)^{8}$ $\mathrm{s}^{-1}$, and the outer boundary energy spectrum, which is an exponential with an $e$-folding energy of $0.1 \mathrm{MeV}$. The diffusion coefficient is consistent with theoretical estimates based on the assumption that diffusion is driven by atmospheric neutral winds.
\end{abstract}

\section{Introduction}

Empirical analyses of radiation belt data traditionally include the extraction of radial diffusion coefficients for comparison with theoretical calculations [Schulz and Lanzerotti, 1974]. While such techniques have been useful for each of the known planetary magnetospheres, they are particularly well suited to the magnetosphere of Neptune, where there is an extended region of stable trapping, from $L \approx 4$ to 14 , with a single dominant and well defined loss mechanism - absorption by the satellite Proteus (1989N1). Radial diffusion of $\geq 1 \mathrm{MeV}$ electrons in the vicinity of the Proteus absorption signature was studied previously [Selesnıck and Stone, 1991b]. Here we extend that analysis to the entire range of available data, compute quantitative estimates of the magnitude and $L$ dependence of the radial diffusion coefficient, and compare the results with expectations based on the assumption that radial diffusion is driven by winds in Neptune's neutral atmosphere.

\section{Empirical Analysis}

The data used in this study are counting rates as a function of time from two solid-state detectors within the electron telescope of the Voyager 2 cosmic ray system (CRS) [Stone et al., 1977]. These detectors (D1 and D2) respond to electrons with kinetic energies $\gtrsim 1$ and $2.5 \mathrm{MeV}$ respectively [Selesnıck and Stone, 1991a,b]. Because only two rates are available in this energy range, they do not provide a unique description of the electron phase space density, $f=j / p^{2}$ where

\section{Copyright 1994 by the American Geophysical Union.}

$j$ is the electron intensity and $p$ the momentum. We therefore use a parameterized model of the intensity to compute a simulated data set, compare it with the real data, and adjust the parameters until a good agreement is obtained. With this approach the non-uniqueness is resolved by the physical constraints of the model, but the validity of these physical constraints must be evaluated independently.

The model is based on radial diffusion conserving the first two adiabatic invariants of particle motion, $M$ and $K$. Steady-state and azimuthal symmetry are assumed, so that $f$ satisfies the diffusion equation [e.g. Schulz and Lanzerotti, 1974]

$$
L^{2} \frac{\partial}{\partial L}\left(\frac{D_{L L}}{L^{2}} \frac{\partial f}{\partial L}\right)=\frac{f}{\tau_{S}}
$$

Electron losses due to absorption by Proteus are included using the lifetime [Selesnick and Stone, 1991b]

$$
\frac{1}{\tau_{S}}=\frac{\Delta t_{S} \omega_{d}}{2 \pi T_{S}}
$$

where $\Delta t_{S}$ is the satellite $L$ shell contact time during one orbital period, $T_{S}$, in the reference frame rotating with the planet (a circular orbit is assumed), and $\omega_{d}$ is the electron drift period. Solutions of (1) at many different values of $M$ and $K$ are combined with the detector response functions, the telescope pointing directions, and the spacecraft trajectory to calculate the two counting rates as a function of time (see Selesnıck and Stone [1991b] for details).

The radial diffusion coefficient has the standard power law form,

$$
D_{L L}=D\left(\frac{L}{5}\right)^{\alpha}
$$

where the parameters $D$ and $\alpha$ are constants to be determined by the data. The model parameterization also includes the choice of boundary conditions for the solutions of the diffusion equation (1). The solutions are relatively insensitive to the inner boundary condition, for which we take $f=0$ at $L=3$, the approximate location of strong absorption by the inner satellites and rings of Neptune. For the outer boundary condition at $L=14$ we have used two different forms, a power law and an exponential in the kinetic energy $E$ :

$$
\begin{gathered}
j(L=14)=A_{0} E^{-\gamma} \\
j(L=14)=A_{0} e^{-E / E_{0}}
\end{gathered}
$$


with parameters $A_{0}$ and $\gamma$ or $E_{0}$ respectively. Note that the boundary conditions do not include any pitch-angle anisotropy. This is because the anisotropy introduced by the conservation of $K$ and the absorption by Proteus are sufficient. However, the data provide only weak constraints on the anisotropy.

The values of the model parameters are varied to minimize the $\chi^{2}$ function

$$
\chi^{2}=\sum_{i=1}^{N}\left(\ln r_{i}-\ln \tilde{r}_{i}\right)^{2}
$$

where $N=433$ is the number of data points, $r_{i}$ is the $i$ th counting rate data point, and $\tilde{r}_{i}$ is the $i$ th simulated data point from the model. The unweighted logarithms of the counting rates are used in $\chi^{2}$ so that all of the data are modeled to approximately the same degree of accuracy. If the counting rates were used instead of their logarithms, then, because of the large range in the data, the model would be significantly constrained only by the highest data values. The same would be true to a lesser extent if the counting rates were weighted by their statistical uncertainties.

The statistical uncertainties in the data are generally small compared to the differences between the model and the data. To obtain an estimate of the model parameter uncertainties we use the standard technique of multiplying the square roots of the diagonal elements of the covariance matrix by $\chi^{2} / \nu$, where $\nu$ is the number of degrees of freedom in the fit. This is equivalent to using the root-mean-square deviations of the model from the data as standard errors on the data points and propagating them into errors on the parameters. In this approach the value of $\chi^{2}$ does not signify the goodnessof-fit because it does not contain the true experimental errors as weighting factors. It does provide a relative measure of the mismatch between the model and the data.

The spacecraft trajectory during the time at which the relevant data were taken is shown in the $R-\Lambda$ magnetic coordinate system [McIlwarn, 1966; see also Selesnick and Stone, 1991b] in Figure 1. Also shown are loci of mirror point locations for particles of constant second adiabatic invariant, $K$, and dipole field lines corresponding to the minimum orbital $L$ shell locations of the satellite Proteus (1989N1). The trajectory is based on the M2 model [Selesnick, 1992] that was developed to account for the location of the outbound Proteus absorption signature, and includes terms of up to eighth order in a spherical harmonic expansion of the planetary magnetic field. This field model was also used in the diffusion calculations.

Simulated data with the real data for comparison are shown for three model fits in Figure 2. The data were chosen to exclude those regions close to Neptune where absorption by the, inner satellites and rings was significant [Stone et al., 1989]. The data have been corrected for background $\left(\sim 25\right.$ counts s${ }^{-1}$ due to penetrating cosmic rays and $\gamma$ rays generated in the spacecraft's power supply) and electronic deadtime ( $20 \mu \mathrm{s}$

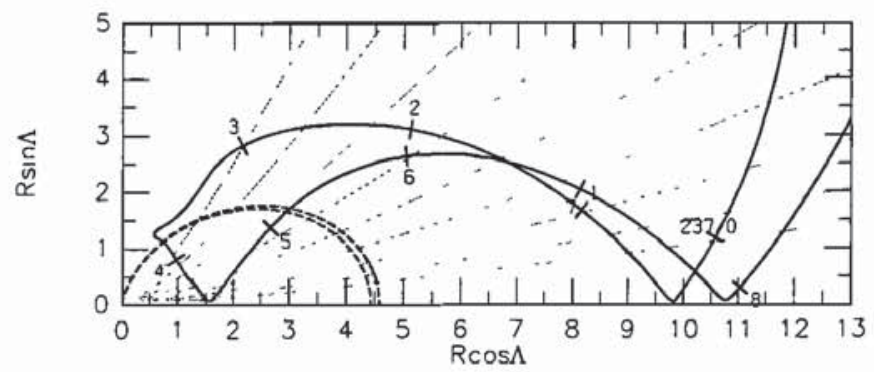

Figure 1. The Voyager 2 trajectory in magnetic coordinates. The tick marks are at 1-hour intervals and are labeled at the beginning of each hour. The dashed dipole field lines correspond to the two orbital minimum $L$ shells reached by Proteus. The approximately radial contours are the mirror point locations of electrons with constant second adiabatic invariant values ranging from $K=0.0125$ to $3.2 \mathrm{G}^{1 / 2} \mathrm{R}_{\mathrm{N}}$ by factors of two.

per count) so that they are linear with respect to the electron intensity. The corrections are only significant near the highest and lowest measured counting rates.

The case with the exponential boundary spectrum (Figure $2 \mathrm{~b}$ ) is clearly a better fit to the data than the power law (Figure 2a). Because the two counting rates could be fit to arbitrary accuracy by either model at a single point in space, this result is indicative of the spatial variations of the energy spectrum, and is consistent with the generally softening spectrum toward lower $L$ shells described by Stone et al. [1989]. A similar result could be obtained with a boundary spectrum consisting of two continuous power laws, but only a marginal decrease in $\chi^{2}$ resulted from the two additional parameters in this model. The best fit to the data provides an estimate of $D_{L L}=5 \times 10^{-8}(L / 5)^{8} \mathrm{~s}^{-1}$, and an $e$-folding energy for the boundary spectrum of $E_{0}=0.1 \mathrm{MeV}$, as indicated in the figure.

The fit in Figure 2c shows the sensitivity of the model to $\alpha$, the radial dependence of $D_{L L}$. The value of $\alpha=$ 3 was fixed and resulted in a significantly higher $\chi^{2}$ than the best fit value of $\alpha=8.1$ in Figure 2b. The significance of this result is discussed below.

Phase space densities, $f(L)$, resulting from the best fit parameters of Figure $2 b$ are shown for selected values of $M$ and $K$ in Figure 3. For each curve only a limited range of $L$ shells are within the energy range to which the two counting rates are sensitive. The figure illustrates the increasing effects of satellite absorption as either $M$ or $K$ increase, as is expected from the lifetime model (2) [Selesnick and Stone, 1991b].

Although the diffusion parameters are well constrained by the data, the agreement between data and model predictions could be improved, suggesting the possible significance of other physical processes. For example, the overall shape of the lower (D2) counting rate is generally more sharply peaked in time (and $L$ ) than the upper (D1) rate and the simulated rate. A possible interpretation is flux limiting due to pitch-angle scattering by whistler mode instability [Kennel and Petschek, 1966]. This would provide an additional loss mechanism for lower energy electrons near their peak 

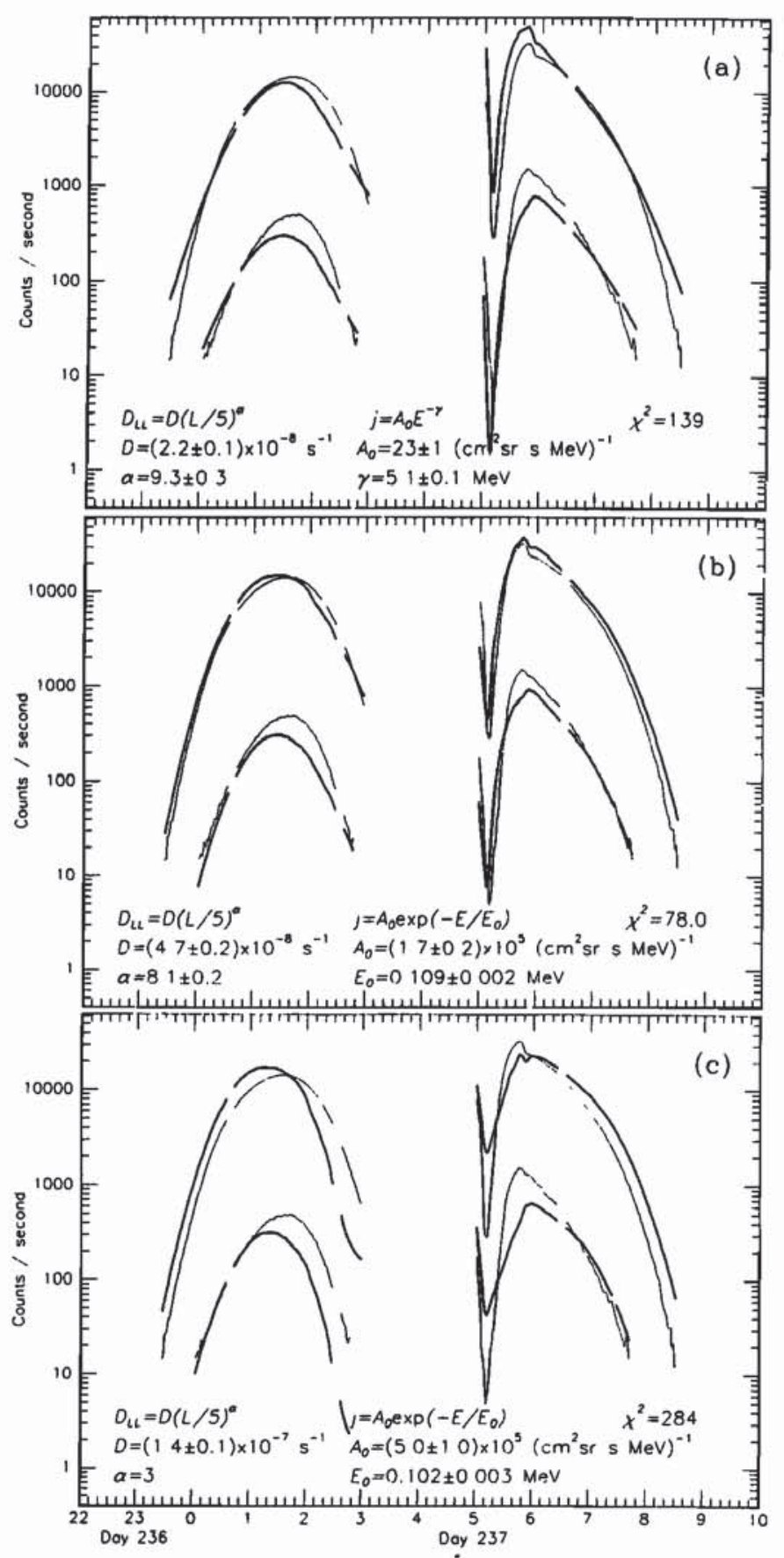

Figure 2. Model predictions (heavy curves) and electron counting rate data (light curves) versus time in hours. The upper and lower traces in each panel are for the D1 and D2 detectors respectively. The model parameter values are also shown in each case.

intensities. Such losses would be fastest for $\sim 10$ to 100 $\mathrm{keV}$ electrons and observations at those energies favor such an interpretation [Krimigis et al., 1990]. The small differences between the data and model predictions in Figure $2 \mathrm{~b}$ suggest that pitch-angle scattering losses may not be significant for electrons $\gtrsim 1 \mathrm{MeV}$. The model also is unable to accurately fit the location of the inbound peak in the lower (D2) counting rate. This may be indicative of remaining uncertainties in the magnetic field model, which lead to substantial uncertainties in the $L$ shell of Voyager 2 on its inbound approach to Neptune [Selesnick, 1992].

\section{Comparison with the Atmospheric Wind Model}

Radial diffusion driven by ionospheric dynamo electric fields due to thermospheric winds was suggested as the dominant transport mechanism for electrons in the radiation belt of Jupiter by Brice and $M c D o n o u g h$ [1973]. Although centrifugally driven transport is probably more important in Jupiter's case outside $L=6$, the atmospheric wind model has been generally accepted for Saturn and Uranus [Hood, 1989]. It is also an attractive model for Neptune because of the high wind speeds (up to $600 \mathrm{~m} / \mathrm{s}$ ) that were observed [Limaye and Sromovsky, 1991].

Electrostatic fluctuations with a spectral density in the $n$th azimuthal harmonic $\tilde{E}_{n}(\omega)$, in resonance with an electron azimuthal drift frequency $\omega_{d}$, lead to a radial diffusion coefficient [Falthammer, 1965; Schulz and Lanzerotti, 1974]

$$
D_{L L}=\frac{1}{8 R_{N}^{2} B_{0}^{2}} L^{6} \sum_{n} n^{2} \tilde{E}_{n}\left(n \omega_{d}\right)
$$

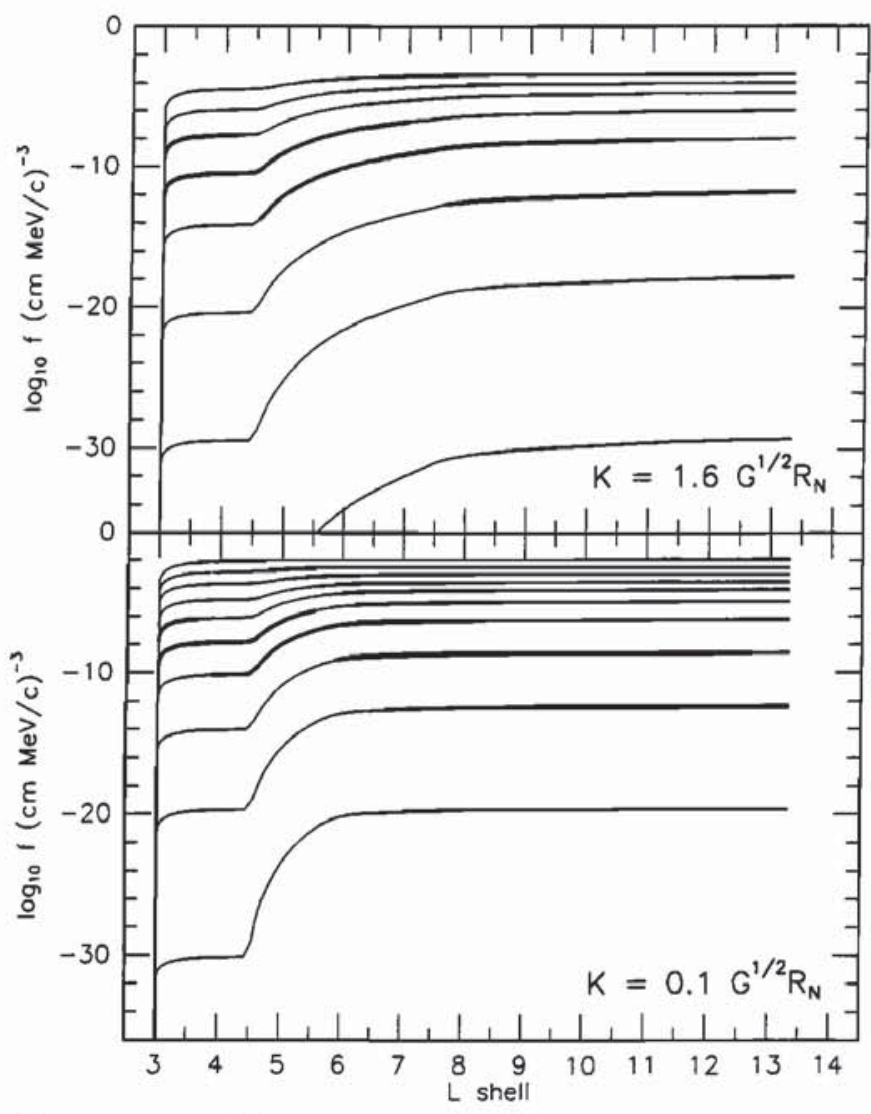

Figure 3. Selected electron phase space densities versus $L$ shell for the best fit model of Figure 2b. The heavy portion of each curve shows where the electrons are in the energy range, from 0.8 to $3 \mathrm{MeV}$, to which the data are sensitive. In each panel first invariant values shown are, from top to bottom, $M=3,10,30,100$, $300,1000,3000,10,000,30,000$, and $100,000 \mathrm{MeV} / \mathrm{G}$. 
where $R_{N}=24765 \mathrm{~km}$ is Neptune's radius and $B_{0}=$ $0.23 \mathrm{G}$ is the surface equatorial magnetic field. Assuming that fluctuations, $\delta W$, in the wind speed occur on the time scale of Neptune's rotational period, $T=16$ hours, we postulate that

$$
\tilde{E}_{1}\left(\omega_{d}\right)=\frac{(2 T / \pi)(\delta E)^{2}}{1+\left(\omega_{d} T / 2 \pi\right)^{2}}
$$

where $\delta E=L^{-3 / 2} B_{0} \delta W$ is the mean electrostatic fluctuation mapped from the ionosphere to the magnetosphere. Then

$$
D_{L L}=\frac{(T / 4 \pi)\left(R_{N} \delta W\right)^{2} L^{3}}{1+\left(\omega_{d} T / 2 \pi\right)^{2}}
$$

Assuming $\delta W=50 \mathrm{~m} / \mathrm{s}$ gives, at low particle energies,

$$
D_{L L} \approx 10^{-6}\left(\frac{L}{5}\right)^{3} \mathrm{~s}^{-1}
$$

which is consistent with the results obtained by Cheng et al. [1992] for $\sim 20$ to $500 \mathrm{keV}$ electrons, and at high particle energies,

$$
D_{L L} \approx 10^{-8}\left(\frac{10^{3} \mathrm{MeV} / \mathrm{G}}{M}\right)^{2}\left(\frac{L}{5}\right)^{7} \mathrm{~s}^{-1}
$$

which is in reasonable agreement with the results obtained above. Note that the assumed form of $\tilde{E}_{1}$ in (7) is appropriate for electrostatic impulses that rise sharply and decay slowly on the drift time scale [Schulz and Lanzerottz, 1974]. Other forms could lead to different $L$ dependences of $D_{L L}$. However, the general agreement between the theoretical and empirical determinations of low $L$ dependence at low energies and high $L$ dependence at high energies lends support to the atmospheric wind driven diffusion model. A similar result for Uranus was obtained by Hood [1989]. We also note, however, that the inclusion of a $M^{-2}$ dependence in $D_{L L}$, as predicted by the theory, does not improve the model fits to the data. This may be due to the limited energy range of the data or to other physical mechanisms that were not taken into account.

Acknowledgments. This work was supported by NASA under contract NAS7-918 and grant NAGW-2402.

\section{References}

Brice, N., and T. R. McDonough, Jupiter's radiation belts, Icarus, 18, 206-219, 1973.

Cheng, A. F., C. G. Maclennan, B. H. Mauk, S. M. Krimigis, and L. J. Lanzerotti, Energetic ion phase space densities in Neptune's magnetosphere, Icarus, 99, 420-429, 1992.

Falthammer, C. G., Effects of time-dependent electric fields on geomagnetically trapped radiation, J. Geophys. Res., 70, 2503-2516, 1965.

Kennel, C. F., and H. E. Petschek, Limit on stably trapped particle fluxes, J. Geophys. Res., 71, 1-28, 1966.

Krimigis, S. M., B. H. Mauk, A. F. Cheng, E. P. Keath, M. Kane, T. P. Armstrong, G. Gloeckler, and L. J. Lanzerotti, Hot plasma parameters in Neptune's magnetosphere, Geophys. Res. Lett., 17, 1685-1688, 1990.

Limaye, S. S., and L. A. Sromovsky, Winds of Neptune: Voyager observations of cloud motions, J. Geophys. Res., 96, 18,941-18,960, 1991.

Mcllwain, C. E, Magnetic coordinates, Space Sci. Rev., 5, $585-598,1966$.

Schulz, M., and L. J. Lanzerotti, Particle Diffusion in the Radiation Belts, Springer, New York, 1974.

Selesnick, R. S., Magnetic field models from energetic particle data at Neptune, J. Geophys. Res., 97, 10,857-10,863, 1992.

Selesnick, R. S., and E. C. Stone, Energetic electrons at Uranus: Bimodal diffusion in a satellite limited radiation belt, J. Geophys. Res., 96, 5651-5665, $1991 a$.

Selesnick, R. S., and E. C. Stone, The electron absorption signature of 1989N1, J. Geophys. Res., 96, 19,137-19,147, $1991 b$.

Stone, E. C., R. E. Vogt, F. B. McDonald, B. J. Teegarden, J. H. Trainer, J. R. Jokipii, and W. R. Webber, Cosmic ray investigation for the Voyager missions: Energetic particle studies in the outer heliosphere-and beyond, Space Sci. Rev., 21, 355, 1977.

Stone, E. C., A. C. Cummings, M. D. Looper, R. S. Selesnick, N. Lal, F. B. McDonald, J. H. Trainor, and D. L. Chenette, Energetic charged particles in the magnetosphere of Neptune, Science, 246, 1489-1494, 1989.

Hood, L. L., Radial diffusion in the Uranian radiation belts: Inferences from satellite absorption loss models, $J$. Geophys. Res., 94, 15,077-15,088, 1989.

R. S. Selesnick and E. C. Stone, California Institute of Technology, mail code 220-47, Pasadena, CA 91125.

(received March 16, 1994; accepted May 12, 1994.) 